\title{
EFECTO AGUDO DEL EJERCICIO FISICO EN LA INTELIGENCIA Y LA MEMORIA EN HOMBRES, SEGUN LA EDAD
}

\author{
Yorleny Alfaro Chavarría y Walter Salazar Rojas \\ Escuela de Educación Física y Deporte \\ Universidad de Costa Rica, San José Costa Rica \\ E-mail:wsalazar@cariari.ucr.ac.cr
}

\begin{abstract}
Resumen
Alfaro, Y. y Salazar, W. (2001). Efecto agudo del ejercicio fîsico en la inteligencia y la memoria en hombres, según la edad. Revista en Ciencias del Ejercicio y la Salud, 1(2), 1-11. Con muy diversas investigaciones se han demostrado los efectos agudos del ejercicio físico en los procesos cognitivos (memoria, inteligencia, tiempo de reacción, creatividad, etc.) según la edad. Sin embargo, gran cantidad de variables se ven involucradas en los diferentes tratamientos. Es, por esto, que se pretendió realizar una investigación específica y homogénea, de manera que los cambios obtenidos en la inteligencia y la memoria en las diferentes edades se deban al tratamiento en sí, y no a la influencia de otros factores. Para esto, se aplicó un tratamiento que consistió en realizar ejercicio aeróbico subiendo y bajando una grada de $27 \mathrm{~cm}$. de alto x $30 \mathrm{~cm}$. de ancho, durante 10 minutos, a una intensidad del 60\% de la frecuencia cardíaca máxima. Los sujetos fueron 100 hombres con edades entre la niñez y la edad avanzada. Se ubicaron en 5 subgrupos que realizaron un "pretest", el tratamiento indicado y un "postest". Las pruebas fueron el test de Raven para la inteligencia y el Verbal Script Digit Span para medir la memoria auditiva. Para conocer los resultados se realizó un análisis de varianza Anova 5x2x2 para grupos independientes, utilizando los puntajes obtenidos en las diferencias entre "pretest" y "postest", para ambas variables, con su respectivo "post-hoc" en los casos requeridos. Y para determinar la magnitud del cambio, se obtuvo los tamaños del efecto. Se encontró que con ejercicio aeróbico submaximal, la totalidad de los grupos etarios mejoraron sus resultados en el test de memoria con mejor resultado los de edades promedio de 25.5 y 63.25 años. Para la variable de inteligencia los sujetos de 34.75 y 52.4 años en promedio lograron los mejores resultados, mientras que los jóvenes de 25.5 años y los adultos mayores no tuvieron cambio significativo en este aspecto. PALABRAS CLAVES: memoria, inteligencia, edad, efecto agudo del ejercicio.
\end{abstract}

\section{INTRODUCCIÓN}

Con el pasar de los años, las investigaciones han podido demostrar que existen cambios positivos en la aptitud física de los individuos que realizan ejercicio físico; gracias a esto, se han abierto nuevos temas de estudio y nuevos retos, al querer determinar si el ejercicio físico influye también en aspectos psicológicos, sociales y cognitivos.

Son investigadores como Brownel, Steen y Wilmore (1987); Horton (1986); Pavlou, Steffe, Lerma y Burrows (1985), quienes demostraron que hay beneficio físico-fisiológico y psicológico debido al ejercicio submaximal, tanto en jóvenes como en adultos.

Otras investigaciones han enfocado el aspecto psicológico; en donde ponen al descubierto los beneficios producidos por el ejercicio, entre ellos: mejorar la autoestima (Hanson y Neede, 1974; Hilyer y Mitchell, 1979); mejorar el estado de ánimo (Berger y Owen, 1983; Blumental y otros, 1982), y disminuir las conductas estresantes (Petruzello, Landers, Hatfield, Kubits y Salazar, 1991; Wilfley y Kunce, 1986).

En la actualidad, el tema del efecto del ejercicio físico en las funciones mentales ha tomado mucho auge; se ha encontrado una gran cantidad de estudios en los que se indica que existe relación entre la condición física y las funciones cognitivas. Son Anthony (1991), Beckman y Danner (1987), Offenbach, Chodzko-zadjo y Ringel (1990), y Young (1979), algunos de los que han encontrado que los sujetos con mejor condición física obtienen mejores resultados en pruebas de memoria, tiempo de reacción y pruebas de razonamiento al compararlo con personas de baja condición física. 
Sin embargo, este tema no sólo se ha estudiado recientemente, ya Gutin (1966); Hart y Shay (1964); Lybrand, Andrews y Ross (1954); comprobaron la influencia del ejercicio físico en las funciones cognitivas y en el rendimiento académico para diversas edades cronológicas.

Estudios realizados sugieren que el ejercicio produce efectos positivos en la habilidad mental, la inteligencia y la memoria de niños con edad escolar (Chisson, 1971; Hart y Shay, 1964; Mero, Jaakola y Komi, 1989; Sanabria, 1995 y Díaz, 1997). Investigaciones con jóvenes, adultos y ancianos demuestran que con ejercicio físico los sujetos han mejorado significativamente las funciones cognitivas (Dustman y otros, 1990; Sanabria, 1995). Otros estudios con ancianos confirman que realizar ejercicio físico permite mejorar su capacidad mental (Rickli y Edwards, 1991; Sabol y Topp, 1990).

Por esta amplia gama de investigaciones y resultados, fue que Sanabria (1995) realizó el metaanálisis sobre "los efectos del ejercicio en parámetros cognitivos", en el cual revela que sí existe un efecto positivo y significativo del ejercicio sobre las funciones cognitivas de inteligencia, memoria, tiempo de reacción y creatividad, y que, aunque la magnitud del cambio varíe de un área a otra, ese cambio va de acuerdo con ciertas condiciones y características que tienen que ver con el tipo de ejercicio, de diseño de investigación y de sujetos en cada estudio.

En este meta-análisis se estudiaron factores como sexo, edad, duración del programa, número de sesiones, duración de la sesión, tipo de actividad e intensidad; esto para cada una de las cuatro áreas cognitivas.

Así, en lo que a la inteligencia se refiere, Sanabria encontró que el efecto del ejercicio varía significativamente en función de la edad del sujeto, siendo mayor el efecto del ejercicio en los sujetos jóvenes de 8 a 17 años y adultos mayores entre los 50 y 90 años. (Ver Figura 1).

Los resultados en el aspecto de memoria, indican que hubo un efecto del ejercicio para todas las categorías de edades estudiadas, es decir, desde los 8 hasta los 90 años, y que es significativamente mayor el efecto del ejercicio sobre la memoria en los sujetos entre los 46 y los 60 años. (Ver Figura 2).
Figura $\mathrm{N}^{\circ} 1$

Efecto agudo del ejercicio físico en la inteligencia según la edad

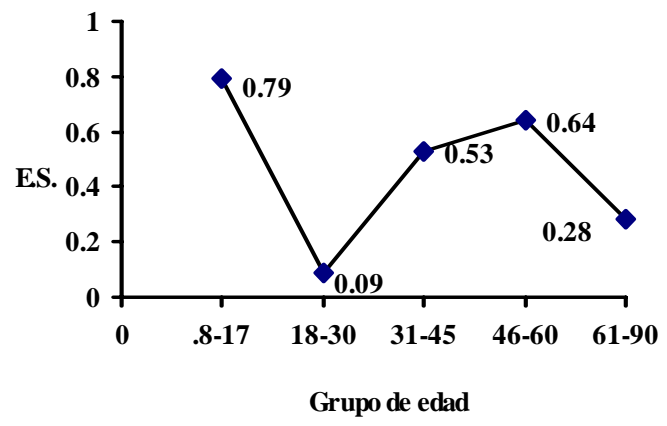

*Tomado de Sanabria (1995)

Figura $\mathrm{N}^{\circ} 2$

Efecto agudo del ejercicio físico en la memoria según la edad

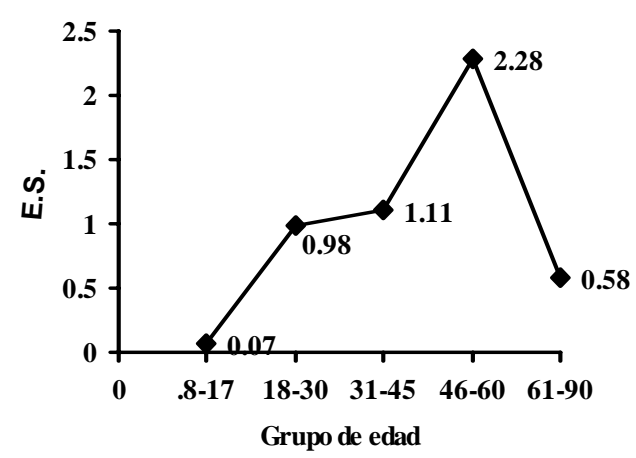

Tomado de Sanabria (1995)

Sanabria (1995), en sus conclusiones enfatiza la importancia de practicar actividades físicas, no sólo para el aspecto físico, sino también para el psicológico e intelectual, y que se debe promover aún más su práctica desde temprana edad hasta edades avanzadas; pues, en diferentes edades, se producen efectos positivos en las actividades cognitivas de los sujetos.

Otros estudios como los de Gutin (1966); Hart y Shay (1964); Lybrand y otros (1954); Merzbacher (1979); Weingarten (1973); Powell (1975); Powell y Pahndolf (1971); Renfrow y Bolton (1979), han probado que el ejercicio físico y 
una mejora en la condición física influyen positivamente en las funciones cognitivas y en el rendimiento académico de niños, jóvenes, adultos y ancianos. Sin embargo, cada uno de estos estudios al igual que el meta-análisis analiza ciertos grupos de edades, pero, ninguno de ellos incluye todas las edades con un mismo tratamiento, de modo que se pueda comparar resultados a través de los diferentes estadios de la vida como tiene por objetivo esta investigación.

Las investigaciones consultadas tienen muy variadas características, tanto en las muestras utilizadas, como los tratamientos aplicados y los diseños de investigación. Igualmente, en el metaanálisis realizado por Sanabria (1995) se utilizaron diversas variables, desde el tipo de investigación y el tipo de intensidad, hasta la duración y el tipo de ejercicio, entre otros aspectos; y aún con esas diferentes variables involucradas se encuentra efecto significativo y positivo del ejercicio físico en los parámetros cognitivos.

Por los resultados obtenidos en esas múltiples investigaciones y en el meta-análisis, es que se hace necesario un estudio confirmatorio con aspectos específicos sobre el tema. Es, por esto, que se pretende realizar esta investigación, la cual consiste en aplicar un programa específico y homogéneo en cuanto a las variables del estudio: las características de la muestra, las características del ejercicio y el tipo de diseño, controlando factores externos y tomando en cuenta, específicamente la variable de la edad, para que así los posibles efectos o cambios se deban a la receptividad de los sujetos al tratamiento.

Por todo lo anterior es que se realizó esta investigación la cual es la primera investigación sistemática que abarca edades desde la niñez hasta el adulto mayor. $\mathrm{Y}$ la primera en utilizar una población netamente masculina, puesto que existen investigaciones de este tipo en mujeres y mixtas, dejando de lado un sector de la población que ahora será retomado.

Las interrogantes planteadas son:

a) ¿causa efecto agudo el ejercicio en la inteligencia?

b) ¿existe efecto agudo por ejercicio en la memoria?. En caso de ser afirmativo, c) ¿cómo varía el efecto del ejercicio en función de la edad?

\section{METODOLOGIA}

\section{Participantes}

La muestra de esta investigación fue de 100 hombres, con edades comprendidas entre los $15 \mathrm{y}$ los 66 años.

Los promedios de edades son indicados en la siguiente tabla:

Tabla $\mathbf{N}^{\circ} 1$

Promedios, desviaciones estándar y rangos para cada grupo de edad estudiado

\begin{tabular}{cc}
\hline & $\begin{array}{c}\text { Edad } \\
\text { Rangos }\end{array}$ \\
\hline $15-17$ & (Promedio \pm desviación estándar) \\
$24-28$ & $16 \pm 0.72$ \\
$33-37$ & $25.5 \pm 1.28$ \\
$50-55$ & $34.75 \pm 1 . .21$ \\
$61-66$ & $52.4 \pm 1.5$ \\
\hline
\end{tabular}

\section{Instrumentos de medición}

Para medir los efectos agudos del ejercicio en la inteligencia y la memoria, se utilizaron dos pruebas. Para la primera variable, fue el test de Raven, con una validez de 0.89 y una confiabilidad de 0.84 (Moreira, 1993), se cataloga como un test de inteligencia general y consta de 60 ítemes de selección única divididos en 5 series de 12 problemas cada uno. Se toma en cuenta el número de errores cometidos, lo que indica que a mayor promedio, menor es el resultado obtenido.

Para la segunda variable se utilizó el Verbal Script Digit Span que se cataloga de memoria auditiva, cuenta con una confiabilidad de 0.79 (Solera, 2000). La prueba tiene dos partes, la parte "A" es la que se utilizó en este caso, consta de 7 niveles, con 2 intentos en cada uno, aquí el evaluador lee al ejecutante una secuencia de números con aproximadamente un segundo de tiempo entre un número y el otro, al finalizar, el sujeto lo repite en el mismo orden que se le dictó. $\mathrm{Si}$ el ejecutante no se equivoca en ninguno de los 
intentos o lo hace sólo en uno de ellos pasa al nivel siguiente. Pero si se equivoca en ambos intentos de un nivel finaliza la prueba y se contabiliza el nivel anterior al que falló. En la parte "B" lo que varía es que al repetir el número dictado el ejecutante lo hace en orden inverso. Por ejemplo se le dicta 691 y él diría 196.

Ambas pruebas fueron aplicadas antes y después del tratamiento y los ítemes seleccionados para "pretest" y "postest" fueron diferentes. En el caso específico del test de Raven, que cuenta con 60 ítemes y con un tiempo de aplicación de 45 minutos, lo que se hizo fue dividirlo en cuatro partes semejantes en dificultad, para ser aplicadas una diferente en cada medición. Así, se utilizaron 15 ítemes en el pretest y 15 en el postest cuando se realizó el ejercicio físico. Los otros 30 ítemes se usaron cuando se realizó la sesión control (sin ejercicio), 15 ítemes para el pretest y 15 ítemes para el postest. El tiempo de aplicación también se redujo a la cuarta parte, o sea se contó con 11 minutos y 15 segundos cada vez.

Con el test de memoria auditiva lo que se hizo fue poner diferentes secuencias de números en cada test, de forma que cada prueba fuera diferente a la anterior, esto para eliminar el factor de aprendizaje que podría alterar los resultados.

Se debe recordar que por tratarse de efectos agudos, fue necesario evaluarlos en un tiempo corto posterior a terminar el tratamiento, debido a que como indica Lamb, (1989) estos son cambios temporales que se producen en el organismo a causa del ejercicio físico.

\section{Procedimientos}

El tratamiento consistió en realizar ejercicio aeróbico subiendo y bajando una grada de $27 \mathrm{~cm}$. de alto $\mathrm{x} 30 \mathrm{~cm}$. de ancho, durante 10 minutos a una intensidad del $60 \%$ de la frecuencia cardíaca máxima.

Se eligió este tratamiento por los resultados obtenidos por Díaz (1997), en donde demostró que con este tipo de actividad física, intensidad y duración se logra un cambio positivo y significativo en la variable de inteligencia.

Para mantener la intensidad de trabajo, se utilizó un metrónomo, con el ritmo asignado previamente, establecido con anterioridad por medio de un estudio piloto. Cada grupo de sujetos trabajó con una misma cadencia, puesto que se utilizó la edad promedio para calcular dicha intensidad.

Cada sujeto participó 2 días diferentes, en donde los grupos con edad promedio de 16 años, 34.75 años y 63.25 años realizaron en la primera sesión el tratamiento propuesto, a la semana siguiente se les midió en reposo (sin ejercicio) mientras que los grupos con edades promedio de 25.5 años y 52.4 años primero fueron medidos en reposo y en la segunda semana se les midió aplicándoles el tratamiento. Esto para ser utilizado como factor de control y poder comparar los resultados obtenidos por los sujetos al hacer ejercicio físico o no hacerlo.

Los sujetos trabajaron de la siguiente manera: se les aplicó el test de memoria auditiva, inmediatamente después el test de Raven, luego de dos minutos se realizó los 10 minutos de ejercicio físico $o$ de reposo según fuera el caso, posteriormente, de nuevo se les aplicó el test de memoria auditiva y el test de Raven.

\section{Análisis estadístico}

En la estadística descriptiva se obtuvieron promedios y desviaciones estándar (D.S.). Para determinar la magnitud del cambio, se calculó los tamaños del efecto (E.S.).

Además se realizó un análisis de varianza de 3 vías $(5 \times 2 \times 2)$, en donde 5 son los niveles de edad; 2 son las mediciones, antes y después del ejercicio y 2 los estados, con o sin tratamiento.

Posteriormente se realizaron los análisis de efectos simples y las pruebas post-hoc en los casos requeridos, esto, para cada una de las variables dependientes: memoria e inteligencia.

También se determinaron los omegas cuadrados $\left(\varpi^{2}\right)$, los cuáles indican el porcentaje de varianza que es debido al tratamiento.

\section{RESULTADOS}

Los resultados obtenidos en este estudio se resumen en la estadística descriptiva que se muestra en la Tabla 2, para la variable de memoria auditiva, 
en el cual se presentan los promedios del pretest, postest con sus respectivas desviaciones estándar, obtenidos por los sujetos de los 5 diferentes grupos de edades.

En la Tabla 3, se resumen de forma semejante los promedios y desviaciones estándar de la variable de inteligencia, la cual fue medida con el test de Raven.
En la Tabla 4, se muestran los cambios de los efectos en las variables de memoria e inteligencia. y las figuras 3 y 4 se facilita observar la magnitud del cambio logrado por el tratamiento según la edad de los sujetos.

TABLA $\mathrm{N}^{\circ} 2$

Promedios, desviaciones estándar y sumatoria de promedios del pretest y postest en la variable de MEMORIA AUDITIVA

\begin{tabular}{cccccccc}
\hline $\begin{array}{c}\text { Condición de } \\
\text { tratamiento }\end{array}$ & medición & $\begin{array}{c}\text { Rango de } \\
15-17 \text { años }\end{array}$ & $\begin{array}{c}\text { Rango de } \\
24-28 \text { años }\end{array}$ & $\begin{array}{c}\text { Rango de 33- } \\
\text { 37 años }\end{array}$ & $\begin{array}{c}\text { Rango de 50-55 } \\
\text { años }\end{array}$ & Rango de 61-66 & Promedios \\
\hline \multirow{2}{*}{ Con ejercicio } & Pretest & $6.25 \pm 0.85$ & $6.6 \pm 0.60$ & $5.95 \pm 0.69$ & $6.05 \pm 0.76$ & $5.45 \pm 0.89$ & 6.06 \\
& & & & & & & \\
Promedios & Postest & $7.10 \pm 0.72$ & $7.5 \pm 0.69$ & $6.7 \pm 0.92$ & $6.6 \pm 0.82$ & $6.7 \pm 0.92$ & 6.92 \\
& & 6.68 & 7.05 & 6.32 & 6.32 & 6.07 & 6 \\
Sin & Pretest & $6.25 \pm 0.64$ & $6.25 \pm 0.79$ & $6.1 \pm 0.85$ & $5.85 \pm 0.93$ & $5.55 \pm 0.76$ & 6 \\
Ejercicio & Postest & $6.4 \pm 0.68$ & $6.6 \pm 1.09$ & $6.2 \pm 1.00$ & $6.1 \pm 1.12$ & $5.6 \pm 0.75$ & 6.18 \\
Promedios & & 6.32 & 6.42 & 6.15 & 5.97 & 5.57 & \\
\hline
\end{tabular}

TABLA $\mathbf{N}^{\circ} 3$

Promedios, desviaciones estándar y sumatoria de promedios del pretest y postest en la variable de INTELIGENCIA

\begin{tabular}{cccccccc}
\hline $\begin{array}{c}\text { Condición de } \\
\text { tratamiento }\end{array}$ & medición & $\begin{array}{c}\text { Rango de } \\
15-17 \text { años }\end{array}$ & $\begin{array}{c}\text { Rango de } \\
24-28 \text { años }\end{array}$ & $\begin{array}{c}\text { Rango de 33- } \\
\text { 37 años }\end{array}$ & $\begin{array}{c}\text { Rango de 50-55 } \\
\text { años }\end{array}$ & Rango de 61-66 & Promedios \\
\hline \multirow{2}{*}{ Con ejercicio } & Pretest & $6.25 \pm 0.85$ & $6.6 \pm 0.60$ & $5.95 \pm 0.69$ & $6.05 \pm 0.76$ & $5.45 \pm 0.89$ & 6.06 \\
& & & & & & & \\
Promedios & Postest & $7.10 \pm 0.72$ & $7.5 \pm 0.69$ & $6.7 \pm 0.92$ & $6.6 \pm 0.82$ & $6.7 \pm 0.92$ & 6.92 \\
& & 6.68 & 7.05 & 6.32 & 6.32 & 6.07 & 6 \\
Sin & Pretest & $6.25 \pm 0.64$ & $6.25 \pm 0.79$ & $6.1 \pm 0.85$ & $5.85 \pm 0.93$ & $5.55 \pm 0.76$ & 6.18 \\
Ejercicio & Postest & $6.4 \pm 0.68$ & $6.6 \pm 1.09$ & $6.2 \pm 1.00$ & $6.1 \pm 1.12$ & $5.6 \pm 0.75$ & 6.57 \\
Promedios & & 6.32 & 6.42 & 6.15 & 5.97 & 5.57 & \\
\hline
\end{tabular}


TABLA N ${ }^{\circ} 4$

Magnitudes del cambio (E.S.) en las variables de MEMORIA AUDITIVA e INTELIGENCIA

\begin{tabular}{ccccc}
\hline & \multicolumn{2}{c}{ Memoria Auditiva } & \multicolumn{2}{c}{ Inteligencia } \\
\cline { 2 - 5 } Rangos de edad & Sin ejercicio & Con ejercicio & Sin ejercicio & Con ejercicio \\
\hline 15-17 años & 0.23 & 1.00 & 0.82 & 1.45 \\
24-28 años & 0.44 & 1.5 & 0.42 & 0.52 \\
33-37 años & 0.12 & 1.09 & 0.66 & -0.33 \\
50-55 años & 0.27 & 0.72 & 0.15 & -0.11 \\
61-66 años & 0.06 & 1.4 & 0.16 & 0 \\
\hline
\end{tabular}

En la Figura 3 se observa que sin ejercicio los sujetos tienen puntajes homogéneos y bajos con magnitudes del efecto (E.S.) menores de 0.5, logrando después del tratamiento un mejor rendimiento que se denota en los E.S. obtenidos, en donde se ve que todos los grupos de edades se benefician con el ejercicio físico (E.S. mayores de 0.72), siendo los grupos de 25.5 y 63.25 años quienes se vieron más favorecidos en lo que a memoria se refiere.

\section{Figura $\mathrm{N}^{\circ} 3$}

Magnitudes del efecto

obtenidas con y sin ejercicio en

la varable de MEMORIA

AUDITIVA

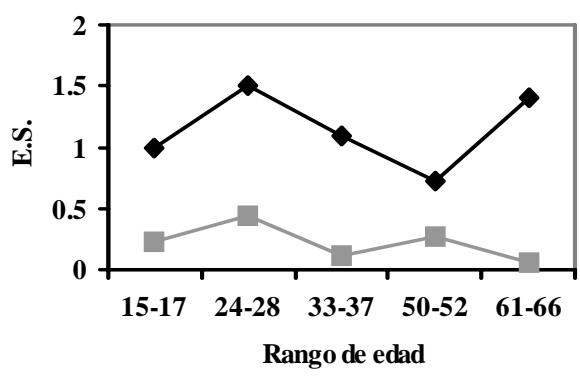

$\sim$ Con ejercicio - Sin ejercico

La Figura 4 muestra que los resultados son bastante heterogéneos, con efectos variados según la edad de los sujetos. Se puede indicar que los adultos de 34.75 años promedio lograron los mejores resultados, mientras que los jóvenes de 25.5 años promedio y los adultos mayores no muestran cambio significativo en lo referente a la inteligencia. Es importante recordar que para esta variable a menor promedio obtenido mejor es el resultado, puesto que los datos denotan el número de errores cometidos por los sujetos.

Los resultados que se muestra en las Tablas 5 y 6 , resumen la estadística inferencial de esta investigación.

En la prueba de ANOVA mixta de $5 \times 2 \times 2$ para la variable de MEMORIA AUDITIVA se encontró que el porcentaje de varianza causada por el tratamiento en los factores es: en edad con un omega cuadrado $\left(\varpi^{2}\right)$ de $8.5 \%$, en ejercicio con un $\varpi^{2}=4.23 \%$, en el aspecto de pretest-postest con un $\varpi^{2}=7.37 \%$, en la interacción doble de pretest-

Figura $\mathrm{N}^{\circ} 4$

Magnitudes del efecto obteni das con y sin ejercicio en la varable de INTELIGENCIA

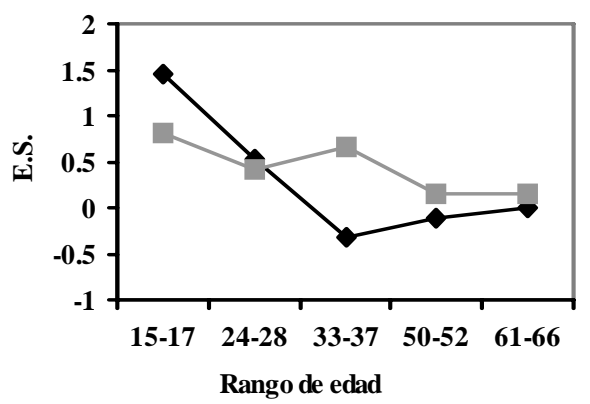

$\multimap-$ Con ejercicio -- Sin ejercico

postest con respecto al ejercicio con un $\varpi^{2}=3.12 \%$ y en la interacción triple de pretestpostest con respecto a ejercicio y a la edad con un $\varpi^{2}=0.39 \%$, el cuál es muy pequeño. (Ver Tabla $6)$. 
Para conocer en dónde se encontraban las diferencias, se realizaron los post-hoc de NewmanKeuls y se encontró que en la variable ejercicio tendieron a obtener mejores resultados en las pruebas de memoria cuando realizaron el tratamiento.

Similares resultados se encontraron en el aspecto de pretest-postest en donde se obtuvieron mejores promedios en el test de memoria al realizar la prueba por segunda vez.
Al analizar las interacciones dobles y descomponerlas en sus efectos simples, se encuentra una diferencia significativa en el aspecto de pretestpostest con respecto al ejercicio y se encuentran mejores resultados en los sujetos al evaluarlos después de haber realizado ejercicio físico.

La interacción triple de pretest-postest con relación al ejercicio y edad fue significativa; $\sin$ embargo su $\varpi^{2}$ es muy pequeño (0.07), lo cual hace que se considere irrelevante su análisis.

TABLA $\mathrm{N}^{\circ} 5$

Estadística inferencial: Anova mixta 5x2x2 en la variable de MEMORIA AUDITIVA

\begin{tabular}{ccccccc}
\hline FUENTE & $\begin{array}{c}\text { Suma de } \\
\text { cuadrados }\end{array}$ & $\begin{array}{c}\text { Grados de } \\
\text { libertad }\end{array}$ & $\begin{array}{c}\text { Cuadrado } \\
\text { medio }\end{array}$ & F & p & $\begin{array}{c}\boldsymbol{w}^{2} \\
(\%)\end{array}$ \\
\hline Edad & 38,635 & 4 & 9,659 & $5,148^{*}$ & 0,001 & 8.5 \\
Error & 178,225 & 95 & 1,876 & & & \\
Ejercicio & 16 & 1 & 16 & $28,057^{*}$ & 0,000 & 4.23 \\
Ejercicio x edad & 2,325 & 4 & 0,581 & 1,019 & 0,401 & 0,01 \\
Error & 54,175 & 95 & 0,570 & & & \\
Pre-postest & 27,04 & 1 & 27,04 & $166,535^{*}$ & 0,000 & 7.37 \\
Pre-post x edad & 1,035 & 4 & 0,259 & 1,594 & 0,182 & 0,11 \\
Error & 15,425 & 95 & 0,162 & & & \\
Pre-post x ejercicio & 11,56 & 1 & 11,56 & $61,783^{*}$ & 0,000 & 3.12 \\
Pre-post x ejercicio x & 2,165 & 4 & 0,541 & $2,893^{*}$ & 0,026 & 0.39 \\
edad & & & & & & \\
Error & 17,775 & 95 & 0,187 & & & \\
\hline
\end{tabular}

TABLA $\mathrm{N}^{\circ} 6$

Estadística inferencial: Anova mixta 5x2x2 en la variable de INTELIGENCIA

\begin{tabular}{ccccccc}
\hline FUENTE & $\begin{array}{c}\text { Suma de } \\
\text { cuadrados }\end{array}$ & $\begin{array}{c}\text { Grados de } \\
\text { libertad }\end{array}$ & $\begin{array}{c}\text { Cuadrado } \\
\text { medio }\end{array}$ & F & p & $\begin{array}{c}\varpi^{2} \\
(\%)\end{array}$ \\
\hline Edad & 124,164 & 4 & 31,041 & $6,501^{*}$ & 0,000 & 4.79 \\
Error & 448,796 & 95 & 4,774 & & & \\
Ejercicio & 176,296 & 1 & 176,296 & $133,631^{*}$ & 0,000 & 7.99 \\
Ejercicio x edad & $32,3,11$ & 4 & 8,078 & $6,123^{*}$ & 0,000 & 1.24 \\
Error & 124,012 & 95 & 1,319 & & & \\
Pre-postest & 23,151 & 1 & 23,151 & $19,007^{*}$ & 0,000 & 1 \\
Pre-post x edad & 38,231 & 4 & 9,558 & $7,847^{*}$ & 0,000 & 1.52 \\
Error & 1114,496 & 95 & 1,218 & & & \\
Pre-post x ejercicio & 1,632 & 1 & 1,632 & 1,557 & 0,215 & 0.03 \\
Pre-post x ejercicio x & 5,792 & 4 & 1,448 & 1,382 & 0,246 & 0.07 \\
edad & & & & & & \\
Error & 98,501 & 95 & 1,048 & & & \\
\hline
\end{tabular}


La variable de INTELIGENCIA también se analizó con una anova de $5 \times 2 \times 2$ y se encontró que el porcentaje de varianza causada por el tratamiento en los factores es: en edad con un omega cuadrado $\left(\varpi^{2}\right)$ de $4.79 \%$, en ejercicio con $\varpi^{2}=7.99 \%$, en el aspecto de pretest-postest con un $\varpi^{2}=1 \%$ y en las interacciones dobles de ejercicio con respecto a la edad un $\varpi^{2}=1.24 \%$ y en la interacción de pretestpostest con relación a la edad un $\varpi^{2}=1.52 \%$, (Ver Tabla 6).

Con respecto al ejercicio, se encontró que fueron aquellos sujetos que realizaron ejercicio físico como lo indica el tratamiento quienes lograron cambios positivos y significativos.

Al analizar la interacción doble para ejercicio con relación a la edad se encontró, según el post-hoc de Newman-Keuls que es a la edad promedio de 34.75 años en donde se logra mayor beneficio en la inteligencia después de realizar el tratamiento.

Otra interacción significativa fue la de pretest-postest con respecto a la edad en donde se encontró resultados similares a la anterior, en donde los sujetos con edad promedio de 34.75 años tendieron a lograr mejores resultados.

En términos generales, se puede indicar que en lo que a memoria se refiere, todos los grupos etarios mejoran sus resultados después de realizar ejercicio físico, especialmente los de edad promedio de 25.5 y 63.25 años. En la variable inteligencia se encuentra que los grupos con edad promedio de 34.75 y 52.4 años de edad son los que tienden a mejorar sus resultados al realizar el tratamiento. Mientras que lo sujetos con edad promedio de 25.5 y 63.4 años no mostraron cambio significativo en este aspecto. Por lo tanto no se puede generalizar, ya que el efecto del ejercicio físico no es contundente para estas edades en la variable de inteligencia.

\section{DISCUSIÓN}

Brownel y otros (1987), Horton (1986), Pavlou y otros (1985) son sólo algunos de los investigadores que han estudiado la influencia del ejercicio físico en el ser humano en los aspectos: físico, social, psicológico y cognitivo, demostrando que se logra beneficio en factores físico-fisiológico y psicológico debido al ejercicio submaximal, en jóvenes y en adultos.

En el aspecto cognitivo específicamente existe variedad de estudios en donde han encontrado una influencia del ejercicio físico con respecto a las funciones cognitivas de memoria e inteligencia para las diversas edades cronológicas como lo afirman desde hace muchos años Gutin (1966), Hart y Shay (1964), Lybrand y otros (1954), y estudios más recientes como el de Sanabria (1995), el de Díaz (1996), y el de Aguirre (2000).

Además, Merzbacher (1979), Weingarten (1973), Powell (1975), Powel y Pahndolf (1971), Renfrow y Bolton (1979), han probado que el ejercicio físico y una mejora en la condición física influyen significativamente en las funciones cognitivas de niños, jóvenes, adultos y ancianos.

Sobre estos cambios Sanabria (1995) da referencias de posibles explicaciones del efecto agudo del ejercicio físico en donde se indica que el ejercicio favorece el transporte y la utilización del oxígeno en el cerebro, el aumento en el metabolismo de la glucosa a nivel celular permitiendo una adecuada provisión y utilización de la energía sin disminuir los niveles de glucosa en el Sistema Nervioso Central y que una mejor renovación de neurotransmisores logran mantener un proceso mental más ágil.

Autores como Landers (1980), Stones y Kozma (1986) indican que otro mecanismo que explica la relación entre ejercicio físico y el aumento en la capacidad cognitiva es la teoría de la activación y de la atención, que plantea que un nivel óptimo de activación permite que los individuos seleccionen y utilicen la información necesaria y de la manera correcta.

Por su parte Chodzko-Zadjo (1991) indica que con el ejercicio físico se aumenta el transporte de oxígeno y nutrientes al cerebro y también se aumenta el flujo sanguíneo permitiendo mejor desempeño, por eso hay cambios en los resultados en las variables cognitivas en las personas independientemente de su edad.

Los estudios sobre la influencia del ejercicio según la edad conllevan a un meta-análisis sobre los efectos del ejercicio en los parámetros cognitivos 
(Sanabria,1995), el cual arroja datos sumamente interesantes en donde indica que en todas las edades los sujetos se ven beneficiados en el aspecto cognitivo, específicamente en la memoria para cualquier edad desde los 8 hasta los 90 años y con mayor efecto entre los 46 y 60 años. En la variable de inteligencia encontró que el efecto varía según la edad de los sujetos donde el mayor efecto fue en jóvenes de 8 a 17 años y en ancianos de 50 a 90 años.

Los hallazgos en las investigaciones del meta-análisis (Sanabria, 1995) son contundentes en resultados, pero abarcan gran cantidad de variables en metodología y tratamientos, por lo cual se realizó este estudio en donde todos los sujetos independientemente de su edad, realizaron el mismo protocolo de ejercicios para que controlando esos factores externos, la variable edad mostrara su influencia en los resultados y se encontró que logra mejorar la memoria auditiva en la totalidad de los grupos de edad estudiados obteniendo mejores resultados los sujetos con edad promedio de $25.5 \mathrm{y}$ 63.25 años.

Al graficar los datos obtenidos en esta investigación para la variable de inteligencia se encuentra un comportamiento en $\mathrm{U}$ de sus resultados, siendo la edad promedio de 34.75 años quienes más se beneficiaron, mientras que en las edades de 25.5 y 63.25 años no mostraron cambio significativo y son los jóvenes de 16 años promedio los que mostraron un detrimento en su rendimiento. Por esta inconsistencia en los resultados es que no se puede generalizar los efectos del ejercicio físico en las diferentes edades para esta variable.

Estos resultados al igual que los obtenidos en otras investigaciones (Sanabria, 1995; Rickli y Edwards,1990 y Sabol y Topp, 1990) demuestran que independientemente de la edad, el ejercicio físico influye en los procesos cognitivos de los sujetos. Aguirre (2000), menciona que con ejercicio puede retrasarse el deterioro y deficiencia que sufren los sistemas psicofísicos a causa del envejecimiento.

Algunos especialistas como Etnier, Salazar, Landers, Petruzello, Han y Nowell (1997) indican que las variaciones en los resultados son porque el ejercicio induce a cambios que involucran el proceso de los neurotransmisores del cerebro en donde se activan sustancias como endorfina, encefalina, serotonina, dopamina y norepinefrina, lo cual es particular en cada sujeto.

Autores como LaForge (1995) afirman que las respuestas psicobiológicas individuales al ejercicio y otros estímulos son invariablemente relacionados al código genético, la naturaleza del ejercicio, el ambiente, la salud, la aptitud y la actitud de los sujetos, por lo tanto el tratamiento(ejercicio) puede causar efectos variados independientemente del grupo etario al que pertenezca, de hecho puede haber variación aún dentro de un mismo grupo de edad.

Parece ser entonces que el tratamiento puede influenciar a los sujetos, pero no se puede esperar que sea de la misma forma en la totalidad de los casos, puesto que algunos de ellos serán más receptivos y lograrán mejores resultados según sea su herencia, motivación e interés.

$\mathrm{Al}$ responder las interrogantes planteadas en este estudio es interesante hacer notar que existe efecto agudo causado por el ejercicio físico en la memoria en todos los grupos de edad estudiados. Con los resultados obtenidos en esta variable se puede decir que con un tratamiento corto y simple se encuentran efectos importantes en los participantes al lograr la combinación adecuada de intensidad, duración y tipo de ejercicio.

Los investigadores mencionan que el ejercicio de corta duración es favorable ya que eleva el nivel de activación a un punto óptimo, lo que permite un rendimiento cognitivo óptimo. (Grupta, Sharma y Jaspal, 1974; Davey, 1972). En cuanto a la intensidad recomiendan la submaximal ya que ha mostrado mejora en las funciones cognitivas de jóvenes, adultos y ancianos tanto a corto como a largo plazo. (Dustman y otros, 1990 y Sabol y Topp, 1990). Y el ejercicio tipo aeróbico, mostró efecto positivo cuando midió en variables cognitivas (Sanabria, 1995 y Díaz, 1997). Todos estos factores se tomaron en cuenta para ser utilizados y probados en este estudio, con lo cual se encontró resultados favorables y beneficiosos en memoria auditiva para los sujetos tratados.

En cuanto a la variable de inteligencia, a pesar de lo concluido por Díaz (1997), para esta investigación en particular se encuentra que el efecto del ejercicio físico varía en función de la 
edad de los sujetos, por lo tanto no se puede recomendar este tipo de tratamiento para todas las edades, debido a que los resultados no son contundentes y sólo los grupos con edad promedio de 34.75 y 52.4 años lograron mejorar sus resultados en el test de Raven.

Una de las cosas más importantes de rescatar en este estudio es que con lapsos cortos de ejercicio físico submaximal se logra beneficio en los aspectos cognitivos de memoria, por lo tanto es recomendable ponerlo en práctica para lograr efectos positivos al menos de corto plazo; queda para futuras investigaciones dar a conocer los cambios crónicos producidos al realizar una actividad física similar. Además, indicar qué efecto se produce según la edad de los sujetos, qué sucede con la población femenina y qué tipo de tratamiento permite que se mejore la inteligencia en los diversos grupos etarios.

\section{REFERENCIAS}

Aguirre, L., (2000). Efectos Crónicos del Entrenamiento de Fuerza sobre Procesos Cognitivos en Personas Mayores de 60 años. Tesis presentada para optar el grado de Licenciatura en Educación Física. Universidad Nacional

Anthony, J. (1991). Psychological aspect of exercise. Clinics in Sports Medicine, 10 (1), 171-180.

Beckman, B.K., y Danner, F. (1987). Effects of

physical conditioning on information - processing efficiency Perceptual and Motor Skills, 65, 175-186.

Berger, B. y Owen, D. (1983). Mood alteration with

swimming: Swimers really do feel better. Psycho-somatic Medicine, 45, 425-433.

Blumenthal, J., William, R. y otros (1982). Psychological changes accompany aerobic exercise in healthy middleage adult. Psychosomatic Medicine, 44, 529-536.

Brownell, K.; Steen, S y Wilmore ,J.(1987). Weight regulation practices in athletes: analisis of metabolics and health effects. Medicine and Science in Sport and Exercise, 19 (6), 546-554.

Chissom,B. (1971). A factor analytic study of the relationship of motor factors to academic criteria for first an third grade boys. Child Development, 42, 1133-1143.

Chodzko-Zajko, W.J. (1991). Physical fitness, cognitive performance and aging. Medicine and science in sport and exercise. 23 (7), 868-872.

Davey, C. (1972). Mental performance after physical activity. Australian Journal of Sports Medicine, 4, 24-33.

Díaz, L. (1996). Efectos agudos del ejercicio en los procesos cognitivos en el niño. Tesis de grado presentada para obtener el grado de Licenciatura en Educación Física. Universidad de Costa Rica.
Dustman y otros. (1990). Age and fitness effects on EEG, ERPs, visual sensitivity and cognition. Neurobiology of Aging, 11, 193-200.

Etnier, J., Salazar,W., Landers, D., Petruzello, S., Han, M., y Nowell, P., (1997). The Influence of Physical Fitness and Exercise Upon Cognitive Functioning: A MetaAnalysis. Journal of Sport / ExercisePsychology, 19, 249-277.

Grupta,V.; Sharma, T. y Jaspal, S. (1974). Physical activity and efficiency mental work. Perceptual and Motor Skill, 38, 205-206.

Gutin,B. (1966). Effect of increase in physical fitness and mental ability following physical and mental stress. Research Quaterly, 37, 211-212.

Hanson, J. y Neede, V. (1974). Longterm physical training effect in sedentary females. Journal of Applied Physiology, 32, 112-116.

Hart, M. and Shay, C. (1964). Relation-ship between physical fitness and academic success. Research Quaterly, 35 (3), 443-445.

Hilyer, J. y Mitchell, W. (1979). Effect of systematic physical fitness training combined with couseling on the selfconcept of college students. Journal of Counseling Psychology, 26, 427-436.

Horton, E. (1986). Metabolic aspects of exercise and weight reduction. Medicine and Science in Sports and Exercise, 18 (1), 10-18.

La Forge, R. (1995). Exercise associated mood alteration: a review of interactive neurobiologic mechanisms. Medicine, exercise, nutrition and health. 4 (1), 17-32.

Lamb, D. (1989). Fisiología del ejercicio: Respuestas y Adaptaciones.(2 ed.), pp 22-28.

Landers, D. (1980). The arousal performance relationship revisited. Research Quaterly for Exercise and Sport, 51 (1), 77-89.

Lybrand, W.; Andrews, T.; y Ross, S. (1954). Sistematic fatigue and perceptual organization. American Journal of Psychology, 67, 704-707.

Merzbacher, C. (1979). A diet and exercise regimen its effects upon mental acuity and personality. A pilot study. Perceptual and motor skill, 48, 367-371.

Mero, A.; Jaakkola, L. y Komi, P. (1989). Neuromuscular, metabolic and hormonal profiles of young tenis players and untrained boys._Journal of Sports Sciencies, 7, 95100

Moreira, R. (1993). Efectos crónicos y agudos del ejercicio en los procesos cognitivos. Tesis presentada para obtener el grado de Licenciatura en Educación Física. Universidad de Costa Rica.

Offenbach, S.; Chodzko-Zajko, W. y Ringel, R. (1990). Relationship between physiological status, cognition and age in adult men. Bulletin of Psychosomatic Society, 28 (2), 112-114.

Pavlou, K.; Steffe, W.; Lerma, R. y Burrows, B. (1985). Effects of dieting and exercise on lean body mass, oxygen uptake and strenght. _Medicine and Science in Sports and Exercise, 17 (3), 466-471. 
Petruzello, S.; Landers, D.; Hatfield, B.; Kubitz, K. y Salazar, W. (1991). A meta- analysis in the anxiety reducing effects of accute and chronics exercise: Outcomes and mechanisms. Sport and Medicine, 11 (3), pp 339- 366.

Powell, R. (1975). Effects of exercise on mental functioning Aims and Perspectives. Journal Sports Medicine, 15, $125-131$

Powell, R. y Pahndolf, R. (1971). Comparison of adult exercisers and non- exercisers on fluid intelligence and select physiological variables. Research Quarterly, 42, 70-77.

Renfrow, N. y Bolton, B. (1979). Personality characteristics associate with aerobic exercise in adult males. Journal of Personality Assessment, 43 (3), 261-266.

Rickli, R. y Edwards, D. (1991). Effects of a three- year exercise program on motor function and cognitive processing speed in older women. Research Quarterly for Exercise and Sport, 62, (1), 61-67.

Sabol, J. y Topp, R. (1990). Effects of moderate and low intensity long term exercise by older adults. Research in Nursing and Health, 13, 209-218.
Sanabria, I. (1995). Meta-análisis sobre los efectos del ejercicio en parámetros cognitivos. Tesis presentada para optar el grado de Licenciatura en Educación Física. Universidad de Costa Rica.

Solera, A. (2000). Efectos de la deshidratación y la rehidratación en variables relacionadas con el rendimiento deportivo. Tesis presentada para optar el grado de Magister Scientiae en Educación Física. Universidad de Costa Rica.

Stones, M. y Kozma, A. (1986). Physical Activity, Age and cognitive-motor performance.

Tomporowski, P.D. y Ellis, R.N. (1986). Effects of exercise on cognitive processes: A review. Psychological Bulletin, 99 (3), 338-346.

Weingarten, G. (1973). Mental performance during physical exertion: The benefits of being physically fit. International Journal of Sport Psychology, 4, 16-26.

Wilfley, D. y Kunce, J. (1986). Differential psychological effects of exercise. Journal of Counseling Psychology, 18 , 459-482.

Young, R. (1979). The effect of regular exercise on cognitive functioning an personality. British Journal Sport Medicine, 13, 110-117. 